\title{
Study on the Regulation of Qinxitong Combined with Methotrexate Tablets on Multiple Inflammatory Indicators and Mechanism Factors in Rheumatoid Arthritis
}

\author{
Zhaoyang Dong1, Jichao Yin ${ }^{2 *}$ \\ 1Shaanxi University of Traditional Chinese Medicine, Xianyang 712046, Shaanxi Province, China \\ 2Xi'an Health School, Xi'an 710000, Shaanxi Province, China \\ *Corresponding author: Jichao Yin, 444604786@qq.com
}

\begin{abstract}
Objective: To study the changes of several inflammatory index mechanism factors in the clinical efficacy of tablet and methotrexate in rheumatoid arthritis. Methods: 20 patients with rheumatoid arthritis were randomly divided into observation $(\mathrm{n}=10)$ and control $(\mathrm{n}=10)$, then normal people as normal $(\mathrm{n}=10)$, all three groups were given methotrexate and the observation group were treated with Qin interest pain tablets. Expression of inflammatory index mechanism factors in each group. Results: After treatment, inflammatory index mechanism factors were detected; a significant decrease of IFN-g, IL-1b, IL-10, IL-37, TNF-a and other factors was found before and after combination treatment (P <0); IL-8 and IL-13 expression $(\mathrm{P}<0)$; the CRP, ESR score of the study group was lower than the control group $(\mathrm{P}<0.05)$; and the inflammatory index mechanism factors were affected by drug combination. Conclusion: Tablets combined with methotrexate in rheumatoid arthritis patients is better than methotrexate. It obviously changed the expression of the patient's inflammatory index mechanistic factors, which has a regulatory effect on the inflammatory mechanistic factors.
\end{abstract}

Keywords: Rheumatoid arthritis; Qin pain; Methotrexate

Publication date: November 2021; Online publication: November 30, 2021

\section{Introduction}

Rheumatoid arthritis (RA) is a systemic autoimmune disease characterized by synovitis as one of the most common chronic inflammatory diseases characterized by inflammatory cell infiltration in joint tissue, synovial hyperplasia, pannus formation and articular cartilage and bone destruction, as well as systemic comorbidities such as lung involvement, vasculitis ${ }^{[1-2]}$. A $50 \%$ risk of developing RA can be attributed to genetic factors ${ }^{[3]}$. Treatment of patients with RA has improved significantly over the past few decades. In particular, therapeutic strategies targeting various cellular and molecular aspects of the immune system, such as B cells, T cell co-stimulation, Janus kinase (Jak) -mediated cytokinin signaling ${ }^{[4-5]}$. Despite these advances, not all patients with RA respond to these therapies or develop resistance to them over time.

The main drug in the treatment of RA is anti-rheumatic drugs (DMARDs), which can reduce synovitis and systemic inflammation and improve function ${ }^{[6]}$. The main DMARD is methotrexate, which can be used in combination with other such classes of drugs. Biologics may be used when rheumatoid arthritis increases or when DMARDs has side effects. Traditional Chinese medicine has significant curative effect on rheumatoid arthritis, and is gradually becoming a first-line drug for the treatment of RA ${ }^{[7-10]}$. In this study, 20 RA patients treated in our hospital were selected to explore the clinical efficacy of pain tablets combined 
with methotrexate on RA patients and test the regulatory effect of inflammatory mechanistic factors.

\section{Materials and methods}

\subsection{General information}

The 20 patients with rheumatoid arthritis and 10 normal people who were treated in our hospital from September 2014 to November 2014 were divided into observation groups, control groups and normal people (10 cases each) according to the random number table method. The sex, age and disease duration of the two general data were not significantly significant. See Table 1.

Table 1. Comparison of General Data of the two groups $(\bar{x} \pm \mathbf{s})$

\begin{tabular}{lccccc}
\hline & Observational group & Control group & Normal group & t or $\boldsymbol{\chi}^{2}$ & $\boldsymbol{P}$ \\
\hline Gender (male / female) & $2 / 8$ & $2 / 8$ & $3 / 7$ & 0.34 & 0.74 \\
Age (year) & $45.8 \pm 7.5$ & $52.6 \pm 5.8$ & $26.8 \pm 5.1$ & 2.32 & 0.055 \\
Disease course (year) & $4.8 \pm 2.6$ & $5.0 \pm 3.9$ & - & 2.46 & 0.185 \\
\hline
\end{tabular}

Inclusion criteria: refer to the 1987 American Society of Rheumatology (ACR) on RA classification criteria, (i) in the bilateral proximal interfinger, palm knuckle, wrist, elbow, meta-toe, ankle, knee joint area, There were lesions in $\geq$ in 3 joint areas; (ii) hand arthritis has joint swelling, May involve the proximal or palm knuckles, wrist involvement; (iii) developed symmetrical arthritis on the left and right sides; The (iv) morning stiffness lasts for at least $1 \mathrm{~h}$; (The above symptoms last at $\geq$ for 6 weeks); (v) developed subcutaneous nodules; Exclusion criteria: (i) serious cardiopulmonary abnormalities; (ii) Bone marrow hematopoietic disorder disease, gastroduodenal ulcer activity period; (iii) is present in pregnant or lactating women;

\subsection{Treatment method}

The control and normal groups were given methotrexate (Shanghai Xinyi Pharmaceutical Company, H31020644) 10mg / time and once / week oral treatment. On this basis, the observation group administered Qin Xipain tablets (homemade Chinese medicine preparations of Xi'an Fifth Hospital, approved No.1570,2001). Both groups were treated for 12 weeks.

\subsection{Index detection}

Refer to the Guidelines for Clinical Research of New Drugs ${ }^{[11]}$. Effective + is effective as the overall efficiency. Effect: all symptoms or main symptoms eliminated, joint function basically recovery; effective: main symptoms basically eliminated, main joint function basically recovery or significant progress; invalid: no progress before treatment. Changes in a number of inflammatory index mechanism factors in the three groups after treatment.

Expression of various inflammatory index factors was examined by qPCR as well as by protein microarray. Protein microarray methods for CRP $(0-8 \mathrm{mg} / \mathrm{L})$, as well as the expression levels of various inflammatory index factors, and ESR $(0-20 \mathrm{~mm} / \mathrm{h}$ for male $0-15 \mathrm{~mm} / \mathrm{h}$, females $)$ by Multiplex ELISA Array.

\subsection{Statistical analysis}

The $\chi^{2}$ was performed using the SPSS18.0 software Inspection and measurement data were indicated by ( $\bar{x}$ $\pm \mathrm{s}), \mathrm{t}$ test for group comparisons and $\mathrm{P}<0.05$. 


\section{Results}

\subsection{Comparison of the clinical efficacy between the two groups}

After treatment, the total efficiency of the observed group was $90 \%$ (9 / 10), significantly higher than the control group by $0 \%(7 / 10)$; the comparison between the two groups was statistically significant $\left(\chi^{2}=\right.$ 4.389, $\quad \mathrm{P}<0.05$ ). See shown in Table 2.

Table 2. Comparison of the clinical efficacy of the two groups (n/\%)

\begin{tabular}{lcccc}
\hline Group & \multicolumn{2}{c}{ Effective } & \multicolumn{2}{c}{ Invalid (it is always efficient) } \\
\hline Observational group & $5(50.0)$ & $4(40.0)$ & $1(10.0)$ & $9(92.5)$ \\
Control group & $4(40.0)$ & $3(30.0)$ & $3(3)$ & $7(72.5)$ \\
$\chi^{2}$ & & & & 4.389 \\
$P$ & & & & 0.024 \\
\hline
\end{tabular}

\subsection{Comparison of CRP and ESR levels between the two patient groups}

No difference was significant between the two CRP, ESR levels before treatment $(\mathrm{P}>0.05)$; the study group had low CRP, ESR levels compared to the control group and were statistically significant $(\mathrm{P}<0.05)$. See Figure 1.
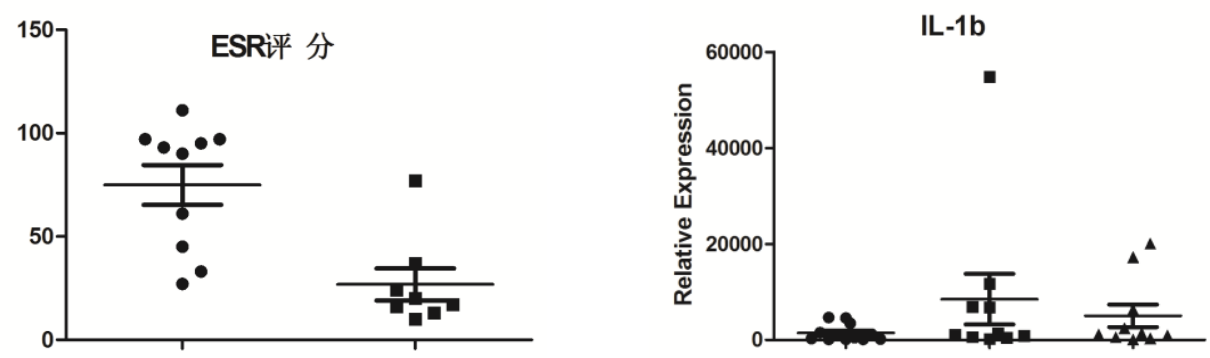

Figure 1. Comparison of CRP and ESR levels between the two groups after treatment

\subsection{Comparison of the expression levels of the inflammatory index factors in each group}

\subsubsection{Factors showed significantly reduced expression before and after drug administration}

A significant reduction in IFN-g, IL-1b, IL-10, IL-37, TNF-a and other factors expression occurred before and after the combination treatment $(\mathrm{P}<0.05)$. See Figure 2, with normal, control and observation groups from left to right.
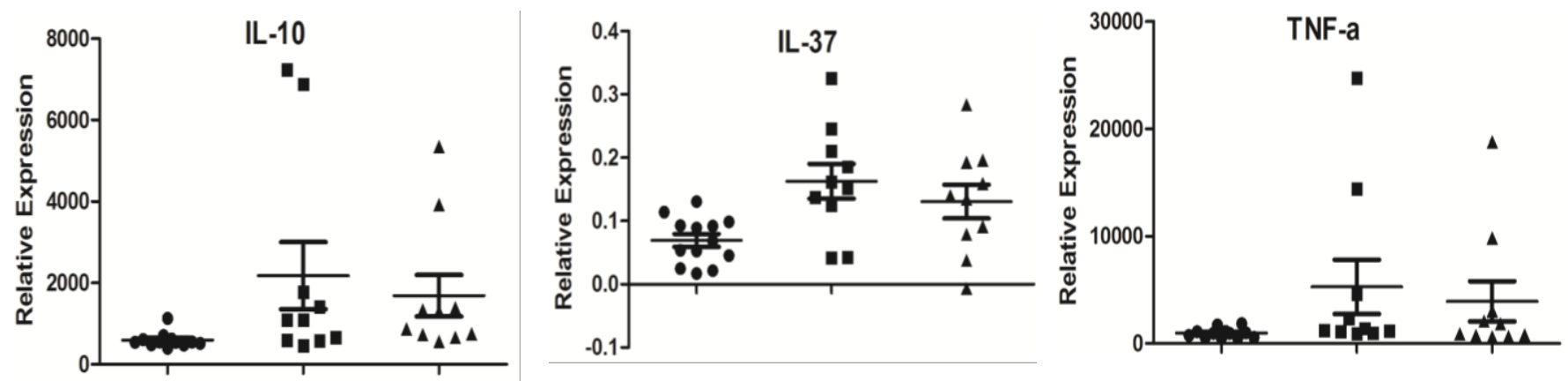

Figure 2. Factors with a significantly reduced expression after treatment 


\subsubsection{Significant increased factors in expression occurred before and after drug administration}

Before and after the combination treatment, both factor IL- 8 and IL-13 factor expression were significantly increased ( $\mathrm{P}<0.05$ ), see Figure 3, with normal groups from left to right, control and observation groups.
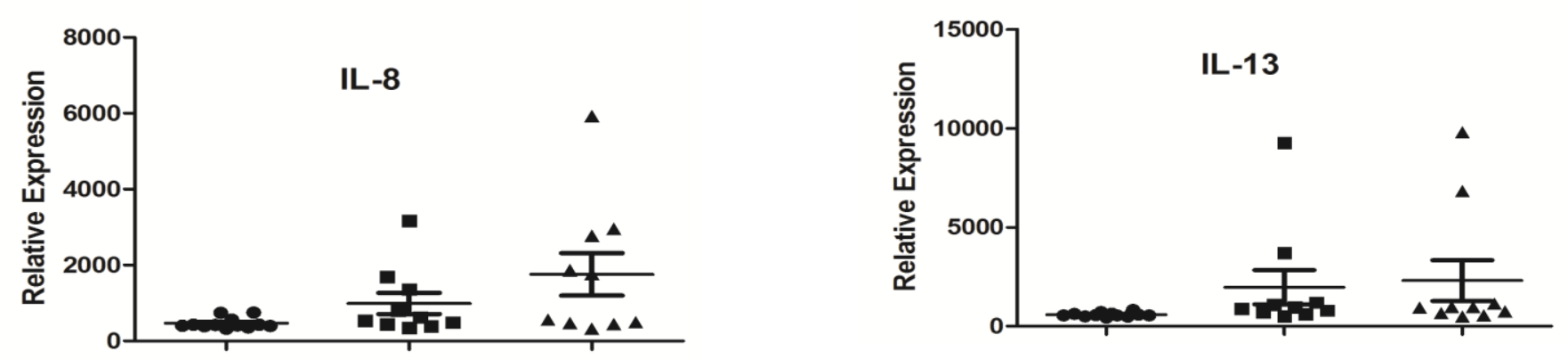

Figure 3. Factors showing a significant increase in expression after treatment

\section{Discussion}

Rheumatoid arthritis (RA) is one of the most prevalent autoimmune diseases in the world. In developed countries, rheumatoid arthritis is 0.5 to $1.0 \%$ in adults. At present, methotrexate is recognized as a well anchored drug for treating RA, but its effect is slow and long-term application is prone to gastrointestinal, liver and kidney function damage. Therefore, most scholars suggest combination medication on this basis to effectively control the progress of the disease and alleviate adverse reactions. This study showed that Qin and methotrexate had significant efficacy in RA patients, and it combined with methotrexate was significantly better than methotrexate monotherapy.

Proinflammatory factors have important roles in the progression of RA, closely related to the occurrence, development, prognosis and disease activity. Thelper cells 17 (Th17) are an important mediator of the immune response against extracellular bacterial and fungal species, and thus play a key regulatory role in the maintenance of mucosal homeostasis ${ }^{[12-13]}$. In contrast, Th17 cells and their effector molecule interleukin 17A, IL-17F, interferon (IFN) g, and tumor necrosis factor (TNF) have been implicated in the pathology of rheumatoid arthritis (RA) ${ }^{[14]}$. The interaction between Th17 cells and other immune cells or stromal cells may eventually lead to chronic inflammation, irreversible cartilage degradation, and bone erosion. Chemokines produced by h17 cells, such as IL17F, IL-22, IL-26, interferon (IFN) g, and tumor necrosis factor (TNF) a, also have important regulatory roles for RA ${ }^{[15-16]}$. This study showed high serum expression levels of IFN-g, IL-1b, IL-10, IL-37, TNF-a and other factors in RA patients with high disease activity, indicating their participation in RA immune inflammatory response; after treatment, serum expression levels of IFN-g, IL-1b, IL-10, IL-37, TNF-a and other factors decreased and reduced disease activity, again confirming that Qin interest pain played an anti-inflammatory role by reducing proinflammatory factor levels in inflammatory tissues.

In conclusion, the clinical effect of RA patients, combined with methotrexate, can significantly improve patient symptoms, and the possible intrinsic mechanism is to reduce the expression levels of serum factors such as IFN-g, IL-1b, IL-10, IL-37, TNF-a. This study provides a new basis for the treatment of RA and for the clinical application of it.

\section{Disclosure statement}

The authors declare no conflict of interest. 


\section{References}

[1] Li Z, 2009, Rheumatoid Arthritis. People's Health Press, Beijing, 6.

[2] Huang Y, Liu Y, Li Z, et al., 2005, Progress in the Factors of Rheumatoid Arthritis. Journal of Difficulties, 4(5): 317-319.

[3] Liu D, Zhang J, Chen Q, et al., 2015, Clinical Application and Research Progress of Autoimmune Antibody Profile in Rheumatoid Arthritis. Chinese Journal of Local Disease Prevention, 30(2): 102103.

[4] Huang B, Wang Q, Liu K, et al., 2013, Relations between TNF-a Signaling and CD4+T Cells in the Development of Rheumatoid Arthritis. Chinese Pharmacology Bulletin, 29(7): 900-903.

[5] Liu D, Liu J, Cao T, et al., 2016, Study on Peripheral Blood CD3+T Lymphocytes and IL-6 Levels in Active Rheumatoid Arthritis. Chinese Journal of Local Disease Prevention and Control, (8): 860-861.

[6] Rheumatology Branch, 2010, Chinese Medical Society. Guidelines for the Diagnosis and Treatment of Rheumatoid Arthritis. Chinese Journal of Rheumatism, 14(4): 265-269.

[7] Jiang Q, Jiang H, Cao W, et al., 2007, Analysis of TCM I in 475 Patients with Rheumatoid Arthritis. Journal of TCM, 48(3): 253-255.

[8] Wan H, 2010, Progress in Chinese and Western Drug Treatment of Rheumatoid Arthritis. Journal of Practical Heart, Brain and Lung Blood Vessels, 18(9): 1379-1380.

[9] Yu S, 2011, Effect of the Effect in 30 Cases of Rheumatoid Arthritis. Sichuan Traditional Chinese Medicine, 29(8): 80.

[10] Huang Y, Ma W, Liu L, et al., 2012, Randomized Controlled Clinical Study of Loose External Application with Miao Medicine and Western Medicine in the Treatment of Acute Cold and Damp Blocked Rheumatoid Arthritis. Practical TCM Internal science, 26(10): 1,2,12.

[11] Zheng X, 2002, Guidelines for Clinical Research of New TCM Drugs. Beijing: China Medical Technology, 115-117.

[12] Nie H, Zheng Y, Li R, et al., 2013, Phosphorylation of FOXP3 Controls Regulatory T Cell Function and is Inhibited by TNF-Alpha in Rheumatoid Arthritis, Nat.Med. 19: 322-328.

[13] Meyer G, Horste Z, Wu C, et al., 2016, RBPJ Controls Development of Pathogenic TH17 Cells by Regulating IL-23 Receptor Expression, Cell Rep, 16: 392e404.

[14] Wu C, Yosef N, Thalhamer T, et al., 2013, Induction of Pathogenic TH17 Cells by Inducible SaltSensing Kinase SGK1, Nature, 496: 513-517.

[15] Wilck N, Matus MG, Kearney SM, et al., 2017, Salt-Responsive Gut Commensal Modulates TH17 Axis and Disease, Nature, 551: 585-589.

[16] Sundstrom B, Johansson I, Rantapaa-Dahlqvist S, 2015, Interaction between Dietary Sodium and Smoking Increases the Risk for Rheumatoid Arthritis: Results from a Nested Case-Control Study. Rheumatology (Oxford), 54: 487-493. 